\title{
LIPID PROFILE IN ESTABLISHED DIABETIC RETINOPATHY- A CASE CONTROL STUDY
}

\author{
Ankita Bhattacharya ${ }^{1}$, Ipsita Mazumdar², Ashok Kumar Maity³ \\ ${ }_{13}^{\text {rd }}$ Year Postgraduate Student, Department of Biochemistry, KPCMCH. \\ ${ }^{2}$ Associate Professor, Department of Biochemistry, KPCMCH. \\ 3Professor and HOD, Department of Ophthalmology, KPCMCH.
}

\begin{abstract}
BACKGROUND
ABSTRACT

In India, diabetes is fast gaining the status of potential epidemic affecting more than 62 million individuals. Diabetic Retinopathy is the commonest microvascular complication prevailing in $18 \%$ of DM population. This study also explores diabetic maculopathy, which is the main cause of vision loss in DR. Epidemiological studies show link between hyperlipidaemia with DR.

Aims and Objectives- To establish the association of hyperlipidaemia with development and graded severity of DR and diabetic maculopathy.
\end{abstract}

\section{MATERIALS AND METHODS}

This case control study was performed between 100 patients of age and gender matched DR as case and 100 patients of diabetes without retinopathy as control, maintaining all inclusion and exclusion criteria at KPC Medical College, Kolkata. The study group was then graded depending on its severity based on ETDRS classification and presence of CSME. Next lipid profile was estimated and compared to find statistical significance. The statistical software SPSS version 20 has been used for the analysis.

\section{RESULTS}

The present study showed statistically significant correlation between diabetic retinopathy and raised total cholesterol level $(p<0.001)$. Increased cholesterol level was significantly associated with the occurrence of all grades of retinopathy. The mean value of total cholesterol in case and control were $215.4 \pm 40.8 \mathrm{mg} / \mathrm{dL}$ and $173.9 \pm 33.8 \mathrm{mg} / \mathrm{dL}$ respectively. The mean total cholesterol was higher in case as compared to control. The mean triglyceride level was also higher in case (165 $\pm 66.5 \mathrm{mg} / \mathrm{dL})$ as compared to control $(135.6 \pm 46.8 \mathrm{mg} / \mathrm{dL})$. This correlation was statistically significant $(\mathrm{p}<0.001)$. In the present study, mean serum cholesterol and LDL concentration was significantly higher in severe DR subjects compared to those with less severe DR. For VLDL and TG, there were no significant differences in the concentration with the severity of DR.

\section{CONCLUSION}

The present study found a significant association between hypercholesterolaemia and CSME ( $p<0.001)$. Except HDL, all mean values of lipid subfractions had significant association with CSME.

\section{KEYWORDS}

Diabetic Retinopathy, Diabetic Maculopathy, CSME.

HOW TO CITE THIS ARTICLE: Bhattacharya A, Mazumdar I, Maity AK. Lipid profile in established diabetic retinopathy- a case control study. J. Evolution Med. Dent. Sci. 2018;7(06):797-800, DOI: 10.14260/jemds/2018/181

\section{BACKGROUND}

Diabetes mellitus is a group of metabolic diseases characterised by hyperglycaemia resulting from defects in insulin secretion, insulin action or both. The chronic hyperglycaemia of diabetes is associated with long-term damage, dysfunction and failure of various organs, especially the eyes, kidneys, nerves, heart and blood vessels. Diabetes causes many complications. Diabetic retinopathy (DR) is the most common microvascular complication in diabetes, which can produce severe visual loss. It is estimated that diabetes mellitus affects $4 \%$ of whole population, almost half of whom have some degree of diabetic retinopathy at a given time.1,2 According to WHO, diabetic retinopathy is responsible for $3 \%-7 \%$ of total blindness in Asia. ${ }^{3}$ In India, the prevalence of diabetic retinopathy in general population is $3.5 \%$ and the

'Financial or Other Competing Interest': None.

Submission 24-11-2017, Peer Review 20-01-2018,

Acceptance 27-01-2018, Published 05-02-2018.

Corresponding Author:

Dr. Ankita Bhattacharya,

AA-218, Sector 1,

Salt Lake, Kolkata-700064.

E-mail: drbhankita@gmail.com

DOI: $10.14260 /$ jemds $/ 2018 / 181$ prevalence of diabetic retinopathy in population with diabetes mellitus is $18 \%{ }^{4}$ In a population-based study in south India, diabetic retinopathy was detected in $1.78 \%$ of diabetic patients screened.5,6

Diabetic retinopathy is primarily classified into nonproliferative DR (NPDR), formally termed simple or background, and proliferative DR (PDR). Progression from mild, characterised by increased vascular permeability to moderate and then to severe and very severe NPDR characterised by vascular closure and increased risk for the development of PDR distinguished by the growth of new blood vessels on the retina and post surface of the vitreous. ${ }^{7}$ Diabetic maculopathy is a form of diabetic retinopathy, in which visual loss occur due to macular oedema. It predominantly occurs in non-insulin dependent diabetics. Diabetic maculopathy can occur at any level of retinopathy and alter the structure of macula, significantly affecting its function. Although, treatment of established retinopathy can reduce the risk of visual loss by $60 \%$. Once diabetic retinopathy or maculopathy occurs there is no satisfactory treatment and the prognosis is very poor, so it is better to prevent its development. Hence, there is need to find out the risk factor associated with DR and CSME (Clinically 
significant macular oedema) and to control the same reduce the incidence of visual loss in future. The present study is taken to establish the relationship of hyperlipidaemia with development and progression of diabetic retinopathy.

\section{MATERIALS AND METHODS}

This case control study was conducted at Department of Biochemistry, KPC Medical College and Hospital, Kolkata for a period of one year. The study included 200 patients. Case group included 100 patients of diabetic retinopathy of $>18$ yrs. of age attending Ophthalmology OPD. This case population was further categorised into 5 subgroups according to ETDRS criteria as follows: 1) Mild NPDR, 2) Moderate NPDR, 3) Severe NPDR, 4) Very severe NPDR and 5) PDR. This population was also subdivided on the basis of Maculopathy (CSME). 100 diabetic patients of Diabetes without diabetic retinopathy > 18 yrs. of age were taken as control. Sample size was taken conveniently.

\section{Inclusion Criteria Included}

1. Patients aged more than 18 years.

2. Patients having other systemic illnesses like hypertension and/or nephropathy and/or hyperlipidaemia.

3. Patients willing to participate in the study.

\section{Exclusion Criteria Included}

1. Patients who have had an episode of chronic inflammatory syndrome, alcoholism or malnutrition will not be included in the study.

2. Patient on diuretics, $\beta$-Blocker, hypolipidaemic agents or any other drug or hormone known to influence lipid or lipid protein metabolism will not be included.

3. Patients having familial hyperlipidaemia.

4. Non-diabetic cases of retinopathy (e.g. infective cause of retinal dystrophy, trauma, toxic maculopathy, ARMD).

5. Subjects not willing for consent.

The study protocol, informed consent and case record form were submitted to the Ethical Committee of K. P. C. Medical College and Hospital for approval.

Informed consent was taken from all participants before inclusion in the study in a language of their own understanding. Illiterate individuals gave their finger-print (left-thumb impression) instead of signature. After obtaining ethical clearance and permission of Head of the Departments of Medicine and Biochemistry and appropriate authority, data collection was started by using pre-designed and pre-tested schedule, interviewing the participants, performing clinical examinations, laboratory investigations and record analysis.

An informed written consent was obtained in every case. A detailed ocular history and medical history was taken. An elaborate biomicroscopic examination of the anterior segment was performed at ophthalmology OPD. Pupils were dilated with topical medication of $1 \%$ tropicamide and 5\% phenylephrine drops, the later being omitted in hypertensives. Detailed fundoscopy was done by direct ophthalmoscopy, indirect ophthalmoscopy and slit lamp biomicroscope using 90D and 70D Volk lens.
After fundus examination, only patients having retinopathy in at least one eye were selected for further study as case and subsequently divided on the basis of grade of DR and presence of maculopathy. Informed consent was taken from the concerned patients. The Diabetic retinopathy was graded according to ETDRS classification.

Fasting venous blood was collected from the antecubital vein of each patient/ study subject in the fasting state (after overnight fasting of 12 hours) in $5 \mathrm{~mL}$ dispensed in clotted vial for estimation of lipid profile. From the clotted blood, serum was separated by centrifugation and stored at -20 degrees centigrade (C).

The collected data were analysed with statistical software SPSS version 20. Data were normally distributed. So we applied independent ' $\mathrm{t}$ ' test and One-Way ANOVA depending upon the number of groups compared. An alpha level of $5 \%$ has been taken, i.e. if any ' $p$ ' value is less than 0.05 it has been considered as significant.

\section{RESULTS}

As per ETDRS classification, Diabetic Retinopathy is divided into NPDR, Moderate NPDR, Severe NPDR, Very Severe NPDR and PDR. Volume of patients in successive order were 40, 33, 12,4 and 11 respectively. Out of 100 Case Study patients, 69 of those were CSME negative and rest 31 subjects were CSME positive.

In the study group, out of 100 patients $60(60 \%)$ were males and $40(40 \%)$ were females. In the control group, 64 (64\%) were males and $36(36 \%)$ were females. There was no significant difference in the gender distribution among the two groups $(\mathrm{p}=0.560)$.

Majority of patients included in this study were in the age group of $51-60$ years (25.5\%). The mean age of the patients in the study group (diabetes with retinopathy) was $60.88 \pm 12.77$ and in control group (diabetes without retinopathy) mean age was $59.63 \pm 13.28$.

This study shows all lipid profile parameters have statistically significant association with development of diabetic retinopathy. When the lipid subfractions in DR subjects with severity of DR were analysed, it was found that mean serum cholesterol and LDL concentration was significantly higher in severe DR subjects compared to those with less severe DR. Also mean serum HDL concentration was higher in early stage DR, but not statistically significant. The present study showed statistically significant correlation between diabetic retinopathy and raised total cholesterol level $(p<0.001)$. Increased cholesterol level was significantly associated with the occurrence of all grades of retinopathy. The mean value of total cholesterol in case and control were $215.4 \pm 40.8 \mathrm{mg} / \mathrm{dL}$ and $173.9 \pm 33.8 \mathrm{mg} / \mathrm{dL}$ respectively. The mean total cholesterol was higher in case as compared to control. The mean triglyceride level was also higher in case $(165 \pm 66.5)$ as compared to control $(135.6 \pm 46.8)$. This correlation was statistically significant $(\mathrm{p}<0.001)$. The present study found a significant association between hypercholesterolaemia and CSME $(p<0.001)$. Except HDL all mean values of lipid subfractions had significant association with CSME. 


\begin{tabular}{|c|c|c|c|c|c|c|c|c|}
\hline & \multicolumn{8}{|c|}{ Group } \\
\hline & \multicolumn{2}{|c|}{ Case } & \multicolumn{2}{|c|}{ Control } & \multicolumn{4}{|c|}{ Results } \\
\hline & Mean & SD & Mean & SD & T-Statistics & $\begin{array}{l}\text { Degrees of } \\
\text { Freedom }\end{array}$ & P Value & Significance \\
\hline HDL & 45.3 & 14.1 & 55.75 & 9.04 & -6.22 & 168 & $<0.001$ & Significant \\
\hline LDL & 136.9 & 32.8 & 90.6 & 32.6 & 10.02 & 197 & $<0.001$ & Significant \\
\hline VLDL & 33.1 & 13.3 & 27.12 & 9.36 & 3.69 & 177 & $<0.001$ & Significant \\
\hline $\mathrm{TG}$ & 166 & 66.5 & 135.6 & 46.8 & 3.74 & 177 & $<0.001$ & Significant \\
\hline CHOL & 215.4 & 40.8 & 173.9 & 33.8 & 7.84 & 191 & $<0.001$ & Significant \\
\hline
\end{tabular}

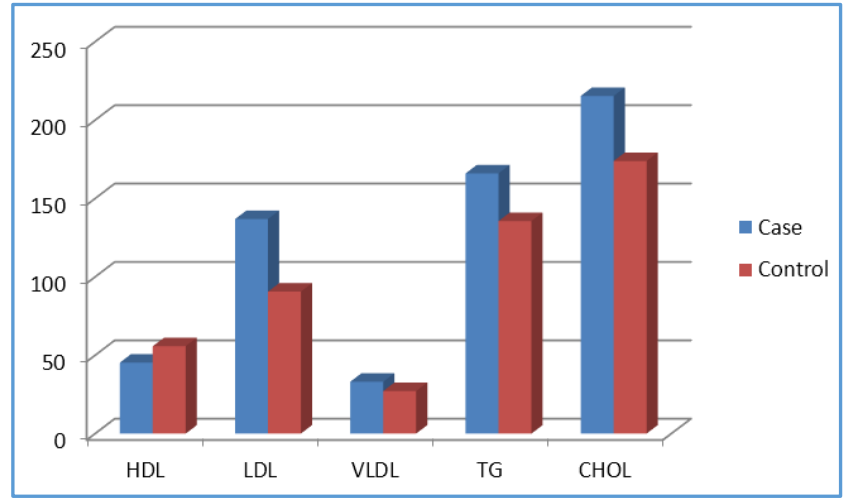

Figure 1. Comparison of Lipid Parameters in Two Groups Studied

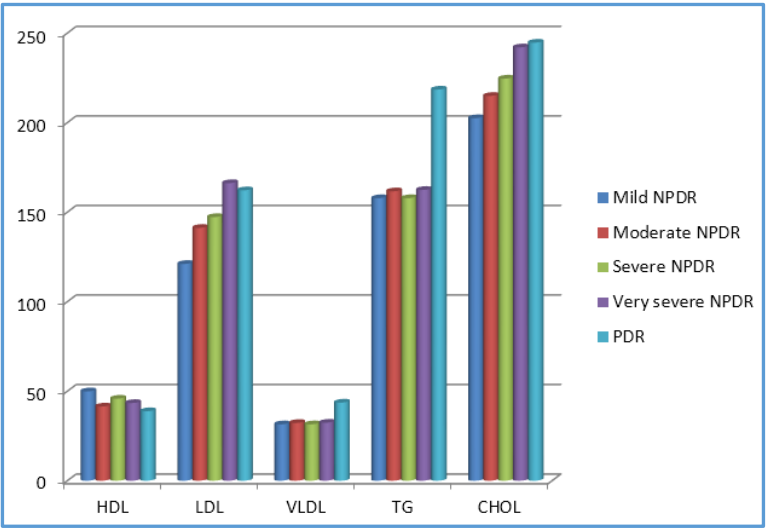

Figure 2. Mean Values of the Lipid Subfractions in Subjects Categorised according to Severity of Diabetic Retinopathy

\begin{tabular}{|c|c|c|c|c|c|c|c|c|c|c|c|c|}
\hline & \multicolumn{10}{|c|}{ Grade } & & \\
\hline & \multicolumn{2}{|c|}{ Mild NPDR } & \multicolumn{2}{|c|}{$\begin{array}{c}\text { Moderate } \\
\text { NPDR }\end{array}$} & \multicolumn{2}{|c|}{ Severe NPDR } & \multicolumn{2}{|c|}{$\begin{array}{c}\text { Very Severe } \\
\text { NPDR } \\
\end{array}$} & \multicolumn{2}{|c|}{ PDR } & & \\
\hline & Mean & SD & Mean & SD & Mean & SD & Mean & SD & Mean & SD & $\begin{array}{c}\mathbf{P} \\
\text { Value }\end{array}$ & Significance \\
\hline HDL & 49.9 & 16.07 & 41.45 & 10.97 & 45.83 & 13.8 & 43.4 & 16.68 & 38.82 & 10.86 & 0.102 & $\begin{array}{c}\text { Not } \\
\text { Significant }\end{array}$ \\
\hline LDL & 121.08 & 33.49 & 141.21 & 30.63 & 147.25 & 20.93 & 166.2 & 16.66 & 162.18 & 26.23 & $<0.001$ & Significant \\
\hline VLDL & 31.5 & 13.37 & 32.24 & 10.85 & 31.5 & 10.17 & 32.4 & 11.46 & 43.64 & 19.4 & 0.481 & $\begin{array}{c}\text { Not } \\
\text { Significant }\end{array}$ \\
\hline $\mathrm{TG}$ & 157.8 & 66.8 & 161.61 & 54.15 & 157.8 & 51 & 162.4 & 56.8 & 218.45 & 96.9 & 0.481 & $\begin{array}{c}\text { Not } \\
\text { Significant }\end{array}$ \\
\hline CHOL & 202.47 & 48.09 & 214.91 & 35.82 & 224.58 & 27.26 & 242 & 13.66 & 244.64 & 22.68 & 0.001 & Significant \\
\hline
\end{tabular}

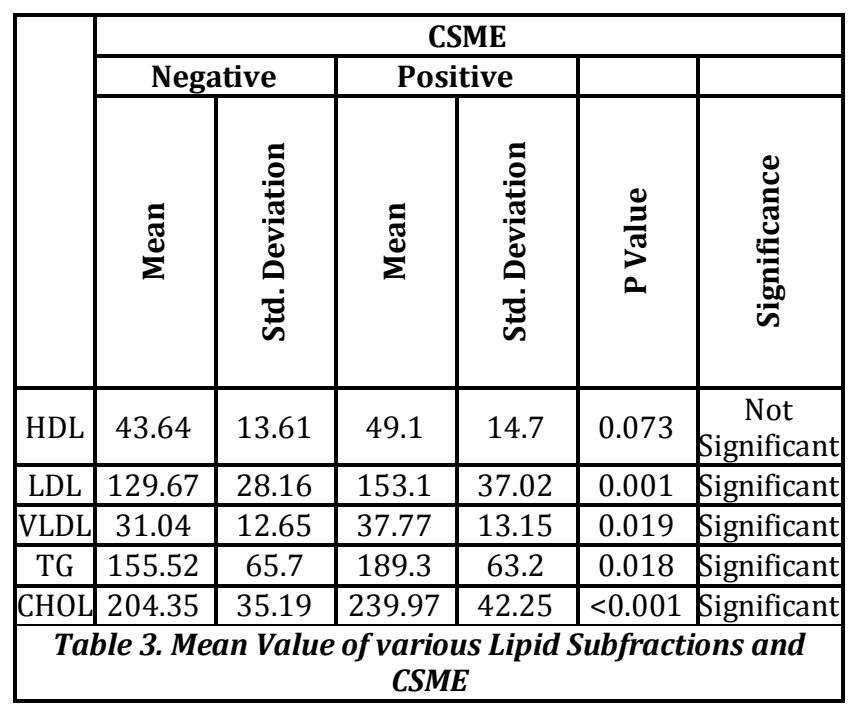

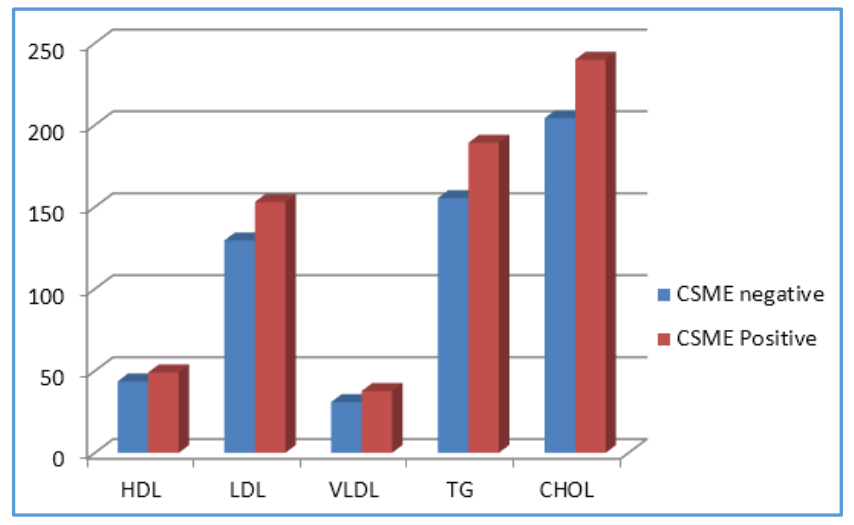

Figure 3. Mean Value of various Lipid Subfractions and CSME 


\section{DISCUSSION}

This study constituted a significant association between hypercholesterolaemia and CSME ( $p<0.001)$. Except HDL, all mean values of lipid subfractions had significant interconnection with CSME. This was in accordance with other studies. Present study shows beyond doubt statistically significant interaction between severity of diabetic retinopathy and raised total cholesterol level ( $p<0.001)$. In case group, mean serum total cholesterol was higher as compared to control. The mean triglyceride level as well was more prominently raised in case collated with control group. This correlation was statistically significant ( $p<0.001$ ). Serum triglyceride $(\mathrm{p}<0.001)$ levels were markedly higher in diabetic retinopathy patients with dissimilarity to those without diabetic retinopathy. These results obtained here are in concordance with the other studies. Mean serum Cholesterol and LDL concentration was remarkably upraised in severe DR subjects compared to those with less severe DR. Correspondingly, mean serum HDL concentration was higher in early stage DR, but did not show any statistical significance. For VLDL and TG, there were no noteworthy differences in the concentrations with the severity of DR.

So, in the present study, an established statistical significance of hyperlipidaemia with diabetic retinopathy and maculopathy was found. The exact reason is not clearly understood. But it has been postulated that elevation of blood viscosity and alterations in the fibrinolytic system occurs in hyperlipidaemia causing hard exudates formation. Chen et al found that dyslipidaemia, not hyperglycaemia induces inflammatory adhesion molecules expression in microvascular human retinal vascular endothelial cells. High lipid levels are known to cause endothelial dysfunction via a local inflammatory response resulting in the release of cytokines and growth factors, activation of oxygen-sensitive biological changes in vessel walls, increased low-density lipoprotein (LDL) oxidation and quenching of nitric oxide. Elevated level of LDL and triglycerides in DM have been linked with higher levels of advanced glycation end products, which play an important role in the pathogenesis of diabetic complications. There may also be incorporation of triglycerides into the cell membrane leading to changes in membrane fluidity and leakage of plasma constituents into the retina. This results in haemorrhage and oedema in the retina. 8,9

\section{Limitation}

The drawbacks of the study are that the fundus photographs which is the standard pattern of recording of fundus changes were not taken for all patients. In such conditions, it is more common to underestimate than to overestimate fundus changes related to diabetic retinopathy. In the present study, CSME was diagnosed by slit lamp biomicroscopy with 78D/ 90D lens. Because of the non-availability, the newer, the more sensitive method of assessing retinal thickening such as optical coherence tomography were not used. As in various other studies, hypertension is an important confounding factor in the study with respect to both diabetic retinopathy and urine albumin excretion. Also, the referral of uncontrolled diabetics to the super-speciality hospital for further management allows the possibility of selection bias to creep into the study. This gives future scope for a populationbased study rather than a hospital-based study.

\section{CONCLUSION}

This discourse has eloquently demonstrated the correlation between diabetic retinopathy and hypercholesterolaemia. Increased cholesterol level was significantly associated with the severity of retinopathy based on ETDRS classification. Much the same illustration is befittingly witnessed for serum LDL. Correspondingly, noteworthy relation was observed between hyperlipidaemia and CSME. These revelations are identical with numerous other studies. Serum triglyceride levels were markedly raised in diabetic retinopathy patients irrespective of its severity. Further studies are mandatory to validate the causal relationship between dyslipidaemia and diabetic retinopathy. Once verified, modification of current treatment guidelines can recommend aggressive lowering of elevated lipids. Among diabetic patients this can lead to depletion of ocular morbidity and associated healthcare costs, thereby potentially improving quality of life.

\section{REFERENCES}

[1] Aiello LP, Gardner TW, King GL, et al. Diabetic retinopathy. Diabetes Care 1998;21(1):143-56.

[2] Wild S, Roglic G, Green A, et al. Global prevalence of diabetes: estimates for the year 2000 and projections for 2030. Diabetes Care 2004;27(5):1047-53.

[3] Meyer JJ, Wang C, Shukla D, et al. Diabetic retinopathy in Asia. Catarct And Refractive Surgery Today 2005:64-8.

[4] Raman R, Rani PK, Rachepalle SR, et al. Prevalence of diabetic retinopathy in India: Sankara Nethralaya diabetic retinopathy epidemiology and molecular genetics study report 2. Ophthalmology 2009;116(2):311-8.

[5] Dandona R, Dandona L, Naduvilath T, et al. Population based assessment of diabetic retinopathy in an urban population in southern India. $\mathrm{Br} \mathrm{J}$ Ophthalmol 1999;83(8):937-40.

[6] Sharma RA. Diabetic eye disease in southern India. J Comm Eye Health 1996;9(20):56-8.

[7] Klein R, Klein BE, Moss SE, et al. The wisconsin epidemiologic study of diabetic retinopathy. II. prevalence and risk of diabetic retinopathy when age at diagnosis is less than 30 years. Arch Ophthalmol 1984;102(4):520-6.

[8] Su DH, Yeo KT. Diabetic retinopathy and serum lipids. Singapore Med J 2000;41(6):295-7.

[9] Goldberg IJ. Clinical review 124: diabetic dyslipidemia: causes and consequences. J Clin Endocrinol Metab 2001;86(3):965-71. 